\title{
Ventilación mecánica en el paciente con lesión cerebral aguda
}

\author{
VINKO TOMICIC F. ${ }^{1}$, MAX ANDRESEN H. ${ }^{2}$
}

'Unidad de Pacientes

Críticos. Clínica Las Lilas. Santiago, Chile, ¿Unidad de Pacientes Críticos, Hospital Clínico Universidad Católica, Departamento de Medicina Intensiva, Facultad de Medicina, Pontificia Universidad Católica de Chile. Santiago, Chile.

Recibido el 8 de septiembre de 2010, aceptado el 29 de noviembre de 2010

Correspondencia: Dr. Vinko Tomicic F. Clínica Las Lilas. Eliodoro Yañez 2087, Sanntiago de Chile. Tel: 56-2-6780997 E-mail:vtomicic@gmail. Unidad de Pacientes Críticos, Hospital Clínico Universidad Católica, Marcoleta 347

E-mail: andresen@med. puc.cl

\section{Mechanical ventilation in patients with acute brain injury}

In about $20 \%$ of patients admitted to an Intensive Care Unit (ICU) the indication of mechanical ventilation $(M V)$ is a neurological disease. These patients have a prolonged $M V$ stay and high mortality. The appropriate use of $M V$ in patients with acute brain injury (ABI) is critical considering that $M V$ by itself is able to induce or worsen an underlying lung injury. Patients with ABI have a higher risk to develop pulmonary complications. During endotracheal intubation the activation of airway reflexes should be prevented, because they may increase intracranial pressure. Tracheostomy is indicated to improve airway management and it is performed in about 33\% of these patients. Indications for MV are loss of spontaneous respiratory effort, changes in lung compliance, gas exchange impairment and ventilatory failure due to muscle fatigue or neuromuscular junction dysfunction. During MV, hypoxemia should be avoided. The $\mathrm{pCO}_{2}$ level has a critical role in cerebral blood flow regulation; therefore a normal $\mathrm{pCO}_{2}$ must be maintained in order to guarantee an optimal cerebral blood flow. Despite that, hypocapnia has been used in patients with increased intracranial pressure, at the present it is not recommended. Its use should be limited to the emergency management of intracranial hypertension, while the underlying cause is being treated. Non-conventional ventilatory modes as prone position ventilation, high-frequency oscillatory ventilation and extracorporeal CO2 removal can be used in patients with ABI. All of them have specific risks and should be employed cautiously. This paper reviews upper airway management and MV in patients with acute brain injury.

(Rev Med Chile 2011; 139: 382-390).

Key words: Brain injuries; Intensive care units; Positive-pressure ventilation.

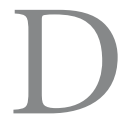
el total de pacientes que requieren ventilación mecánica (VM) en unidades de cuidados intensivos (UCI) alrededor de $20 \%$ corresponden a patologías neurológicas ${ }^{1}$. Este grupo de pacientes presenta una estadía en VM más prolongada y mayor mortalidad ${ }^{2}$.

Considerando que la VM por sí misma es capaz de inducir o agravar el compromiso pulmonar subyacente y que los pacientes con lesión cerebral aguda (LCA) presentan mayor riesgo de desarrollar neumonía por aspiración, edema pulmonar neurogénico, lesión pulmonar aguda y síndrome de distrés respiratorio agudo (LPA/SDRA), es fundamental el empleo apropiado de la $\mathrm{VM}^{3}$.

En la etapa inicial del manejo ventilatorio de un paciente con LCA es clave la intubación endotraqueal (IET), ya que la activación de reflejos de la vía aérea pueden incrementar la presión intracraneal (PIC). Esto es especialmente importante cuando la distensibilidad cerebral se encuentra extremadamente reducida, requiriéndose conexión a VM mediante sedo-analgesia óptima. Así se logra el acoplamiento del paciente al ventilador en particular bajo presencia de patrones ventilatorios 
anormales: respiración de Biot, Cheyne-Stokes, atáxica, etc.

Además de evitar la hipoxemia $\left(\mathrm{PaO}_{2}<60\right.$ $\mathrm{mmHg}$ o $\mathrm{SaO}_{2}<85 \%$ ), elemento asociado a mal pronóstico en la $\mathrm{LCA}^{4}$, debemos procurar un valor de presión parcial de $\mathrm{CO}_{2}\left(\mathrm{PaCO}_{2}\right)$ que asegure un flujo sanguíneo cerebral (FSC) óptimo, controlando gases en sangre arterial 20 a 30 minutos post-conexión para evaluar $\mathrm{PaCO}_{2}{ }^{5}$.

La VM puede afectar la hemodinámica cerebral en forma directa o mecánica, debido al incremento de la presión intratorácica, por uso de elevada presión positiva al final de espiración (PEEP), alto volumen corriente, auto-PEEP, hipertensión intraabdominal, etc., o indirecta o metabólica: aquellos provocados por modificaciones de las presiones parciales de los gases arteriales secundarios a la programación del ventilador ${ }^{5}$.

El objetivo de este trabajo es describir aspectos relacionados con el manejo de la vía aérea superior, de la VM y sus consecuencias, haciendo especial énfasis en aquellos casos en que la LCA se encuentra acompañada de insuficiencia respiratoria aguda (IRA). Considerando la incorporación cada vez más frecuente de nuevas estrategias de VM como: ventilación en decúbito prono (VDP), ventilación con alta frecuencia oscilatoria (VAFO) y el concepto de pulmón abierto (open lung concept), nos referiremos al rol de estas técnicas en estos pacientes.

\section{Intubación endotraqueal}

La IET está indicada para proteger al paciente de una aspiración pulmonar o cuando existe obstrucción de la vía aérea superior; sin embargo, la IET también puede ser necesaria en casos donde la ventilación no parece ser un problema inminente: compromiso de conciencia moderado u oscilante, disfunción bulbar u obstrucción mecánica por caída de la lengua hacia la pared posterior de la faringe (floppy airway) ${ }^{6}$. Los pacientes cuyo puntaje de Glasgow (GCS) sea $<10$ deberían considerarse en riesgo de desarrollar complicaciones en su vía aérea ${ }^{7}$.

La IET debe efectuarse con especial cuidado en enfermos con potenciales lesiones cervicales, ya que la extensión de cuello puede agravar una lesión medular. En presencia de lesiones a nivel de C5 o superiores debemos intubar en forma inmediata tomando en cuenta la alta asociación con íleo y aspiración bronquial secundaria. Lesiones sobre C5, requerirán probablemente VM prolongada y traqueostomía debido a la parálisis de los nervios intercostales y frénicos ${ }^{8}$. Lesiones bajo C 5 también pueden llegar a presentar paro respiratorio a pesar de aparentar una función ventilatoria normal. Esto último es común y puede ocurrir entre la primera y segunda semana post injuria. Se atribuye a fatiga muscular, manejo inefectivo de las secreciones, atelectasias y neumonía. En esta etapa el apoyo con kinesiterapia es crucial ${ }^{9}$.

La intubación guiada con fibroscopía es una excelente alternativa en casos complejos. Otro aspecto esencial de la IET, en pacientes con LCA, es evitar la hipotensión arterial (presión sistólica < $90 \mathrm{~mm} \mathrm{Hg}$ ) e hipoxemia, ya que de ocurrir, la mortalidad se duplica ${ }^{10}$. Ambas consideraciones son claves para evitar la injuria cerebral secundaria.

La inducción anestésica durante la IET debe ser equilibrada para evitar la activación de los reflejos de la vía aérea, hipotensión y desaturación. Se recomienda el uso de bloqueantes neuromusculares no despolarizantes tanto para la IET como durante la VM (Tabla 1).

Tabla 1. Efectos neurofisiológicos de las drogas utilizadas para intubación y sedación

\begin{tabular}{|lcc|}
\hline Agentes & FSC & PIC \\
Benzodiacepinas & $\downarrow$ & $\downarrow$ \\
Opiáceos & $\leftrightarrow$ & $\leftrightarrow$ \\
Barbitúricos & $\downarrow \downarrow$ & $\downarrow \downarrow$ \\
Propofol & $\downarrow \downarrow$ & $\downarrow \downarrow$ \\
Dexmedetomidina & $\downarrow$ & $\downarrow$ \\
Ketamina & $\uparrow \uparrow$ & $\uparrow *$ \\
Etomidato & $\downarrow$ & $\downarrow$ \\
Rocuronio & $\leftrightarrow$ & $\leftrightarrow$ \\
Succinilcolina & $?$ & $\uparrow$ \\
\hline
\end{tabular}

Gremmelt A, Braun U. Analgesia and sedation in patients with head-brain trauma. Anaesthesist 1995; 44 Suppl 3: S559-65.

FSC: flujo sanguíneo cerebral. PIC: presión intracraneal. * No afectaría la hemodinámica cerebral con uso previo de midazolam. 


\section{Traqueostomía}

La traqueostomía (TQT) se realiza en alrededor de 33\% de los pacientes neurocríticos, ya que facilita el manejo de la vía aérea, especialmente en pacientes con lesiones infratentoriales. Basados en el alto porcentaje de reintubaciones se recomienda sea efectuada en el octavo día. Sin embargo, los criterios de selección son controvertidos.

Es así que en pacientes neurocríticos, al comparar traqueostomía precoz ( $<9$ días) con traqueostomía tardía ( $>9$ días), la primera acorta el tiempo de VM y reduce los requerimientos de sedación, incidencia de neumonía asociada a VM, uso de antibióticos y la estadía en UCI. La decisión de practicar TQT, debería basarse en el pronóstico neurológico, conocimiento de la técnica, riesgos de la intubación prolongada, costo del proceso, práctica local y comunicación con la familia ${ }^{11}$.

\section{Indicaciones de ventilación mecánica}

La indicación de $\mathrm{VM}$ en estos pacientes es multifactorial. La decisión de conectar a cualquier paciente crítico a VM es clínica y debe ser tomada con la urgencia que amerita cada caso en particular, incluso sin esperar resultados de laboratorio. Para quienes recién se incorporan al trabajo en UCI neurológicas o neuro-quirúrgicas, existen algunos parámetros que pueden ser de utilidad. (Tabla 2).

Las indicaciones de VM pueden ser categorizadas en cuatro grupos: 1) pérdida del esfuerzo respiratorio espontáneo; 2) alteraciones de la distensibilidad pulmonar; 3 ) deterioro del intercambio de gases y 4) insuficiencia ventilatoria por fatiga muscular o disfunción de la placa neu-

Tabla 2. Indicadores clínicos y de laboratorio de IET y VM

1. Frecuencia respiratoria $<6$ ó $>30$ ciclos por minuto
2. Uso de musculatura accesoria
3. Respiración paradojal
4. Episodios de apnea
5. $\mathrm{PaO}_{2}<60 \mathrm{~mm} \mathrm{Hg}$
6. $\mathrm{PaCO}_{2}>50 \mathrm{~mm} \mathrm{Hg}$

IET: intubación endotraqueal. VM: ventilación mecánica. romuscular. Los pacientes comatosos desarrollan frecuentemente patrones respiratorios anormales que pueden llegar a tener implicaciones clínicas.

Las principales metas de la VM son: sostener o modular, según sea el caso, el patrón de ventilación, corregir la hipoxemia y evitar la acidosis respiratoria, factores que juegan un rol crítico en los pacientes con cualquier grado de LCA.

En ciertas circunstancias debemos anticiparnos al deterioro neurológico, por ejemplo, en la hemorragia subaracnoídea aneurismática que cursa con vasoespasmo cerebral, la mejor estrategia puede ser la IET y conexión a VM con el fin de asegurar el intercambio de gases, ya que el paciente se encuentra recibiendo terapia enérgica con fluidos, lo que podría causar edema pulmonar ${ }^{12}$.

\section{Efectos hemodinámicos y metabólicos de la VM}

\section{Rol de la $\mathrm{PaCO}$ en la hemodinámica y el metabolismo cerebral}

Mientras la $\mathrm{PaCO}_{2}$ se mantenga en rangos fisiológicos, la relación entre ésta y el flujo sanguíneo cerebral (FSC) es prácticamente lineal. $\mathrm{La} \mathrm{PaO}_{2}$, en cambio, interfiere con el FSC sólo en condiciones de hipoxemia grave; en pacientes con distensibilidad cerebral reducida, las modificaciones del volumen sanguíneo cerebral (VSC) se acompañan de cambios simultáneos de la presión intracraneana (PIC), relación que ha sido extensamente utilizada como herramienta terapéutica ${ }^{13}$.

$\mathrm{La} \mathrm{PaCO}_{2}$ es un poderoso modulador del FSC. Por una parte, la hipercapnia se asocia con vasodilatación y por lo tanto con incremento del VSC y del FSC, factores que en presencia de LCA son capaces de aumentar la PIC y producir herniación del tronco encefálico. La alcalosis hipocápnica, al incrementar el pH del líquido cefalorraquídeo (LCR), es capaz de producir vasoconstricción cerebral, reducción del flujo sanguíneo cerebral (2 $\mathrm{mL} /$ minuto por cada $\mathrm{mm} \mathrm{Hg}$ que cae la $\mathrm{PaCO}_{2}$ ) y por consiguiente disminución de la $\mathrm{PIC}^{14}$. Cabe destacar que los vasos sanguíneos cerebrales son más sensibles a los cambios de $\mathrm{pH}$ del LCR que a las modificaciones de la $\mathrm{PaCO}_{2}$ o del bicarbonato. El rango en el cual el cambio de la $\mathrm{PaCO}_{2}$ posee mayor impacto sobre el calibre de los vasos cerebrales es entre 20 y $60 \mathrm{mmHg}$; en este intervalo el FSC se modifica $3 \%$ por cada $\mathrm{mmHg}$ que varía la $\mathrm{PaCO}_{2}{ }^{15}$. 
Los cambios inducidos por la hipocapnia pueden desviar la curva de autorregulación del FSC hacia la derecha. Sin embargo, sus efectos vasoconstrictores son transitorios, ya que el $\mathrm{pH}$ extracelular cerebral, gracias a mecanismos compensadores (anhidrasa carbónica de los plexos coroideos y excreción de bicarbonato a nivel renal), tiende a normalizarse en pocas horas, motivo por el cual el efecto inicial se pierde luego de 6 a 12 horas de iniciada la hiperventilación ${ }^{16}$. Debemos recordar que la discontinuación de la hiperventilación puede producir vasodilatación de rebote con el incremento subsecuente de la $\mathrm{PIC}^{17}$.

En la etapa inicial del TEC, debido a la marcada reducción del FSC (global y regional), la hiperventilación podría precipitar isquemia cerebral y empeorar los desenlaces clínicos. El Cambridge Neurocritical Care Group demostró que la hiperventilación aumenta el riesgo de isquemia cerebral en pacientes con TEC y que los cambios isquémicos pueden ser inadvertidos si se utilizan sistemas de monitorización global de la oxigenación cerebral, como la saturación venosa del bulbo de la yugular $\left(\mathrm{SjvO}_{2}\right)^{18,19}$. Episodios de hiperventilación periódica reducen en forma concomitante la presión tisular de oxígeno cerebral $\left(\mathrm{PtiO}_{2}\right)$ y el FSC (cambios en espejo), los cuales se hacen más marcados si son repetidos en el tiempo ${ }^{20}$.

El mayor estudio realizado hasta la fecha en pacientes con TEC grave, comparó en forma prospectiva pacientes manejados con normoventilación $\left(\mathrm{PaCO}_{2} 35 \pm 2 \mathrm{mmHg}\right.$ ), hiperventilación $\left(\mathrm{PaCO}_{2} 25 \pm 2 \mathrm{mmHg}\right)$ e hiperventilación más trometamina. Concluyó que quienes recibieron hiperventilación presentaron peor desenlace neurológico a los 3 y 6 meses $^{21}$. En el ambiente pre-hospitalario, la $\mathrm{PaCO}_{2}$ se correlaciona con mayor mortalidad hospitalaria. Así, pacientes que ingresan con $\mathrm{PaCO}_{2}<35 \mathrm{mmHg}$, la mortalidad fue $77 \%$, con hipercapnia $61 \%$ y con normocapnia de $15 \%{ }^{22}$.

En presencia de una disminución del FSC en pacientes con LCA en las primeras horas de evolución, se hace recomendable evitar la hiperventilación. Considerando los complejos cambios hemodinámicos y metabólicos a los que está sometido el cerebro lesionado, es aconsejable implementar neuromonitorización multimodal para adecuar estrategias de protección cerebral y minimizar la injuria cerebral secundaria, entre ellas la programación de la VM.
Resumiendo, la hiperventilación o mejor dicho la hipocapnia ha sido una práctica habitual en pacientes con LCA que presentan hipertensión intracraneal (HIC), no obstante, por sus serios efectos adversos no se recomienda, limitándose exclusivamente al tratamiento de HIC en casos de emergencia mientras se trata la causa subyacente $^{23,24}$.

\section{Efectos de la PEEP en paciente neurocrítico}

El manejo ideal de la LCA y de la HIC ha incluido tradicionalmente una estrategia dirigida a la protección de la vía aérea, optimización de la entrega de oxígeno al cerebro, control estricto de la $\mathrm{PaCO}_{2}$ y limitación de los efectos adversos que podría tener la VM con presión positiva sobre la PIC. Históricamente, dichas metas se conseguían con el uso de elevado volumen corriente $\left(\mathrm{V}_{\mathrm{T}}\right)$, altas concentraciones de $\mathrm{O}_{2}$, niveles bajos de PEEP y fluidos más vasopresores para mantener una presión de perfusión cerebral (PPC) adecuada.

Si bien la VM puede contribuir a alcanzar dichas metas, hoy sabemos que una inapropiada programación de los parámetros ventilatorios $\left(\mathrm{V}_{\mathrm{T}}\right.$ y PEEP) puede inducir o agravar las alteraciones del parénquima pulmonar, proceso conocido como daño pulmonar inducido por el ventilador

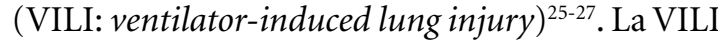
se vincula con dos fenómenos: el primero, ocurre al final de la inspiración y se asocia con el uso de $V_{T}$ o presión inspiratoria elevados capaces de inducir sobre-distensión alveolar (volu-barotrauma) ${ }^{28,29}$. El segundo, ocurre al final de la espiración e inicio de inspiración siguiente (tidal recruitment) y se relaciona con la aplicación de un nivel de PEEP insuficiente para evitar el colapso-reapertura alveolar cíclico (atelectrauma) ${ }^{30,31}$. La consecuencia biológica de la transgresión de estos límites generará liberación de mediadores pro-inflamatorios, reclutamiento de leucocitos, activación y propagación del proceso inflamatorio (biotrauma), factores responsables del desarrollo de disfunción orgánica múltiple ${ }^{32,33}$.

Si consideramos que la bóveda craneana es una caja cerrada que contiene estructuras compresibles (cerebro, LCR y compartimiento vascular), cuya curva presión-volumen presenta un comportamiento no lineal, la adición o retiro de cualquiera de sus componentes generará cambios correspondientes en la presión del sistema. En este sentido, 
el continuo drenaje de sangre a través de los vasos venosos yugulares es esencial para poder recibir simultáneamente la sangre arterial, evitando el estasis circulatorio cerebral.

En consecuencia, podemos decir que hay obstáculos teóricos considerables para implementar una estrategia de VM protectora en pacientes con LCA. Por una parte, la adición de PEEP podría teóricamente disminuir la PPC, por aumento de la presión intratorácica, la cual puede obstaculizar el retorno venoso desde el cerebro (aumento de la presión venosa yugular) hacia la aurícula derecha, incrementar el VSC $y$, por ende, reducir la distensibilidad cerebral, aumentando la PIC ${ }^{34}$. Por la otra, elevados niveles de PEEP podrían mejorar la oxigenación al prevenir o revertir el colapso alveolar en zonas dependientes, fenómenos frecuentes en este tipo de pacientes. En consecuencia, el uso de PEEP es al menos controversial: corrige la hipoxemia y limita el desarrollo de VILI y por otro lado puede llegar a reducir la PPC.

Estudios en pacientes con LCA y LPA/SDRA han reportado que la PIC se incrementa significativamente cuando la aplicación de PEEP se asocia con aumentos de la $\mathrm{PaCO}_{2}$ (sobredistensión alveolar) y ésta no varia significativamente cuando la PEEP conduce a un aumento de la distensibilidad pulmonar y disminución de la $\mathrm{PaCO}_{2}$ (reclutamiento alveolar) ${ }^{35}$. Por tanto, los efectos de la PEEP dependen muy probablemente de la mecánica pulmonar y del potencial para reclutamiento individual de los pacientes, lo que explicaría los efectos opuestos de su aplicación. Otra forma de predecir el impacto de la PEEP sobre la presión arterial media, PPC y PIC es midiendo la distensibilidad del sistema respiratorio $\left(\mathrm{D}_{\mathrm{SR}}\right)$, así los efectos adversos de la PEEP serán más prominentes en aquellos pacientes cuya $D_{S R}$ es normal que en aquellos con una $\mathrm{D}_{\mathrm{SR}}$ baja $^{36}$.

Estudios en animales expuestos a LCA traumática masiva y VM agresiva $\left(\mathrm{V}_{\mathrm{T}}\right.$ elevado y PEEP bajo), presentaron mayor edema y daño pulmonar que aquellos sin $\mathrm{LCA}^{37}$. Un estudio multicéntrico en pacientes con LCA demostró que el uso de $\mathrm{V}_{\mathrm{T}}$ elevado fue un factor predictivo independiente de desarrollo de LPA/SDRA. Este estudio provee antecedentes que respaldan el uso de estrategias de VM protectoras en estos pacientes ${ }^{38}$. Sin embargo, existe controversia para recomendar su amplio uso, ya que el $\mathrm{V}_{\mathrm{T}}$ bajo se asocia con reducciones importantes del volumen minuto y con el desarrollo de hipercapnia. En el mismo sentido, niveles elevados de PEEP obligan a reducir el $\mathrm{V}_{\mathrm{T}}$ y/o la presión de driving (presión meseta menos PEEP) para evitar la transgresión de la presión de distensión (meseta $\leq 30 \mathrm{~cm} \mathrm{H}_{2} \mathrm{O}$ ).

A pesar que los estudios disponibles sugieren que la estrategia de VM protectora basada exclusivamente en la reducción del $\mathrm{V}_{\mathrm{T}}$ puede llevarse a cabo sin llegar a la hipercapnia ${ }^{39}$, los niveles elevados de PEEP son bien tolerados en la mayoría de los pacientes con $\mathrm{LCA}^{40}$.

En la mayor parte de los casos el uso de PEEP incrementará el volumen de fin de espiración, previniendo el colapso alveolar y reclutando aquellas unidades previamente colapsadas. Este mecanismo reduce el cortocircuito intrapulmonar y mejora la oxigenación, efectos benéficos tanto en el parénquima cerebral como para el resto de la economía.

\section{Manejo ventilatorio del paciente neurocrítico con LPA/SDRA}

La incidencia de LPA/SDRA en pacientes con LCA aislada fluctúa entre 20 y $25 \%$, asociándose con mayor riesgo de permanecer en estado vegetativo persistente y fallecer. Los factores de riesgo más relevantes para predecir su desarrollo son: TAC de cerebro alterado y GCS bajo al ingreso ${ }^{41-43}$.

El manejo ventilatorio de los pacientes con LPA/SDRA se basa en la presencia de un pulmón que posee escasa capacidad de aireación (pulmón pequeño o baby lung) obligando a utilizar una estrategia de ventilación protectora. Como se discutió, esta estrategia genera preocupación tanto por sus efectos hemodinámicos como en la ventilación alveolar. En dos de los estudios más importantes publicados en los últimos años, ARDS Network y el ALVEOLI, la media de $\mathrm{PaCO}_{2}$ en el primer día de VM alcanzó valores entre 40 y $41 \mathrm{mmHg}$ respectivamente, lo que sugiere que la VILI puede prevenirse en la mayoría de los casos, sin incrementar los niveles de $\mathrm{PaCO}_{2}$. No obstante, algunos pacientes desarrollaron aumento progresivo de la $\mathrm{PaCO}_{2}$ secundario a hipoventilación alveolar ${ }^{39,44}$.

En este escenario es preciso realizar maniobras que permitan optimizar la eliminación del $\mathrm{CO}_{2}$, como: disminuir el espacio muerto del circuito ventilatorio (uso de filtros HME pediátricos o sistemas de humidificación activa), mejorar la sincronía paciente-ventilador, descartar obstrucciones del TET y considerar maniobras para mejorar 
la $\mathrm{D}_{\mathrm{SR}}$ como drenaje de líquido ascítico o pleural. $\mathrm{Si}$ a pesar de ello, la $\mathrm{PaCO}_{2}$ continúa elevada, se debe evaluar caso a caso la utilidad de limitar el $V_{T}$, diferenciando claramente aquellos pacientes que están evolucionando con LPA/SDRA versus aquellos que están con ventilación mecánica protectora profiláctica ${ }^{45}$. En tal caso se recomienda aumentar el volumen corriente a $8 \mathrm{ml} / \mathrm{kg}$ de peso ideal, sin que signifique necesariamente incrementar el riesgo de desarrollar VILI ${ }^{38}$.

En un estudio multicéntrico, realizado por nuestro grupo, en 19 UCI de Chile, los pacientes cuyo motivo de inicio de la VM fue coma (de diversas causas), el $V_{T}$ fue mayor y la PEEP menor que en aquellos que ingresaron por insuficiencia respiratoria aguda, sin que esto haya significado diferencias significativas en la mortalidad ${ }^{46}$. Cabe destacar que no todos los pacientes que ingresaron a VM por trastornos neurológicos presentaban LPA/SDRA.

Con el objetivo de evitar la injuria secundaria en pacientes con LCA que cursan con LPA/SDRA se recomienda la implementación de neuromonitoreo multimodal, lo que permite medir en forma directa los cambios de la $\mathrm{PaCO}_{2}$ y su correlación con la hemodinámica y metabolismo cerebral (PIC, PPC, $\mathrm{SjvO}_{2}, \mathrm{PtiO}_{2}$ ), permitiendo un manejo más seguro e integral.

\section{Ventilación mecánica no convencional en paciente con LCA}

\section{Ventilación en decúbito prono (VDP).}

Los pacientes que cursan con LPA/SDRA ventilados en posición supina desarrollan atelectasias en las áreas dependientes del pulmón, deteriorando la oxigenación y generando alteraciones en la relación ventilación perfusión $(\mathrm{V} / \mathrm{Q})^{47}$. La VDP, en cambio, mejora la oxigenación, previene el desarrollo de atelectasias optimizando la relación $\mathrm{V} / \mathrm{Q}^{46}$. Un meta-análisis reciente mostró disminución de la mortalidad en el grupo de pacientes con SDRA grave sometidos a VDP ${ }^{49}$.

Pocos estudios han evaluado la VDP en pacientes con LCA asociada a LPA/SDRA.Un estudio aleatorio realizado en pacientes en coma, en VM, demostró una disminución del puntaje de injuria pulmonar (LIS: lung injury score) y de la frecuencia de neumonía asociada a ventilación mecánica en el grupo en VDP. Sin embargo, este grupo presentó mayores niveles de PIC respecto de los ventilados en posición supina ${ }^{50}$.

Análisis retrospectivos de pacientes con HSA y SDRA asociaron la VDP con aumentos significativos de la oxigenación arterial y cerebral, pero con aumentos de la PIC y disminución de la $\mathrm{PPC}^{51}$. Lo anterior es opuesto a los hallazgos de otro estudio en pacientes con TEC y hemorragia intracerebral más SDRA, el cual mostró que la VDP no tuvo efectos a nivel de la PIC ni PPC, mejorando en forma significativa la oxigenación y la distensibilidad del sistema respiratorio ${ }^{52}$. Tabla 3.

\section{Ventilación oscilatoria de alta frecuencia}

La ventilación con alta frecuencia oscilatoria (VAFO) se caracteriza por una combinación de presión media de vía aérea elevada $(25-35 \mathrm{~cm}$ $\mathrm{H}_{2} \mathrm{O}$ ), volumen corriente inferior al espacio muerto de la vía aérea $(2-4 \mathrm{ml} / \mathrm{kg})$, administrados con una frecuencia respiratoria suprafisiológica (5-10 $\mathrm{Hz}$ ). Usado en el escenario neonatal y pediátrico este método ha sido recientemente introducido como estrategia ventilatoria en pacientes adultos con LPA/SDRA ${ }^{53}$.

Las ventajas de la VAFO sobre los modos convencionales de VM son su capacidad para prevenir

Tabla 3. Ventilación Mecánica Invasiva y LCA

Protección de la vía aérea, normocapnia y oxigenación adecuada

LPA/SDRA

Ventilación en decúbito prono

(VDP)

Ventilación con alta frecuencia oscilatoria (VAFO)
VM protectora ( $\leq 8 \mathrm{ml} / \mathrm{kg}$ de peso ideal) evitar sobredistensión alveolar, volumen minuto necesario para $\mathrm{PaCO}_{2}$ entre 30 y $35 \mathrm{~mm} \mathrm{Hg}$, PEEP suficiente para evitar atelectrauma Evaluación de la distensibilidad, PEEP según lo anterior e individualizado para cada paciente, nomocapnia

Controversial, pacientes con LPA/SDRA ventilados que no mejoran pese a optimización de la VM en posición supino, idealmente contar con neuromonitorización multimodal

Controversial, debe ser efectuada en pacientes con neuromonitorización multimodal

LPA/SDRA: Lesión Pulmonar Aguda/Síndrome de Distrés Respiratorio Agudo. LCA: Lesión Cerebral Aguda. 
el de-reclutamiento alveolar y limitar la sobredistensión alveolar de manera simultánea, debido a que las oscilaciones se restringen a la zona segura de la curva volumen-presión del sistema respiratorio. Esta técnica sería tan segura y efectiva para mejorar la oxigenación en SDRA grave como la VM convencional ${ }^{54,55}$.

Las aplicaciones potenciales de la VAFO en pacientes con LCA/SDRA han sido pobremente evaluadas. Salim comunicó mejoría de la oxigenación y ventilación acompañada de una disminución de la PIC ${ }^{56}$. David, empleando VAFO, observó mejoría de la $\mathrm{PaO}_{2}$ con aumento de la PIC y caída de la PPC, pero esto ocurrió en una minoría de los $\operatorname{casos}^{57}$. En su estudio recomendó que el uso de esta técnica en pacientes con LCA debe ser efectuada con neuromonitorización ${ }^{57}$. Un estudio reciente en 38 pacientes con TEC e insuficiencia respiratoria hipoxémica mostró una modesta, pero significativa, reducción de la PIC con la técnica de alta frecuencia por percusión ${ }^{58}$ (Tabla 3 ).

La VAFO permanece todavía como una terapia de rescate en pacientes con SDRA y LCA grave. La experiencia en estos casos es limitada, por lo tanto, debe monitorizarse de cerca para hacer las rectificaciones oportunamente.

Respecto de sistemas que permiten remoción de $\mathrm{CO}_{2}$, es fundamental tener cautela en caso de implementarla en presencia de LCA, dado los riesgos de la anticoagulación. Con oxigenación con membrana extracorpórea (ECMO) hay solamente reporte de casos, por tanto su uso es excepcional (terapia de rescate) y debe ser empleada con mínima anticoagulación o con circuitos recubiertos con heparina para impedir la hemorragia cerebral. Existen también casos exitosos donde se ha asociado hipercapnia permisiva con remoción extracorpórea sin bomba ${ }^{59}$.

\section{Conclusiones}

Los pacientes que cursan con LCA asociada a LPA/SDRA constituyen una subpoblación única que obliga a controlar múltiples aspectos, tales como: protección de la vía aérea, optimización de la entrega de oxígeno al cerebro, titulación estricta de la $\mathrm{PaCO}_{2}$ y limitación de los efectos adversos que podría tener la $\mathrm{VM}$ con presión positiva sobre la PIC. Clásicamente la hiperventilación fue considerada una práctica habitual en pacientes que cursaban con HIC; sin embargo, por sus efectos adversos serios, actualmente no se recomienda su uso rutinario, limitándose exclusivamente al tratamiento de la HIC en casos de emergencia mientras se trata la causa subyacente.

El uso de $V_{T}$ elevado ha demostrado ser un factor predictivo independiente de desarrollo de LPA/SDRA en pacientes con LCA, aspecto que respalda el uso de estrategias de VM protectoras. Si bien la hipercapnia es una de las consecuencias más temidas, hay estudios que muestran que este efecto no se presenta en todos los pacientes.

Con el objetivo de evitar el daño secundario en los pacientes con LCA que cursan con LPA/SDRA se recomienda la implementación de neuromonitoreo multimodal. De esta manera es posible medir, al lado de la cama del paciente, los cambios de la $\mathrm{PaCO}_{2}$ y su correlación con la hemodinámica y metabolismo cerebral (PIC, $\mathrm{PPC}, \mathrm{SjvO}_{2}, \mathrm{PtiO}_{2}$ ) con el fin de ofrecer un manejo más seguro e integral en este tipo de pacientes.

\section{Referencias}

1. Esteban A, Anzueto A, Frutos F, Alía I, Brochard L, Stewart T E, et al. Characteristics and outcomes in adult patients receiving mechanical ventilation: a 28 -day international study. JAMA 2002; 287: 345-55.

2. Esteban A, Anzueto A, Alia I, Gordo F, Apezteguía C, Pálizas F, et al. How is mechanical ventilation employed in the intensive care unit? An international utilization review. Am J Respir Crit Care Med 2000; 161: 1450-8.

3. Stevens R, Lazaridis C, Chalela J. The Role of Mechanical Ventilation in Acute Brain Injury. Neurol Clin 2008; 26: 543-63.

4. McHugh GS, Engel DC, Butcher I, Steyerberg EW, Lu J, Mushkudiani N, et al. Prognostic value of secondary insults in traumatic brain injury: results from the IMPACT Study.Journal of Neurotrauma 2007: 24: 287-93.

5. Belda FJ, Aguilar G, Soro M, Maruenda A. Ventilatory management of the severely brain-injured patient. Rev. Esp. Anestesiol. Reanim. 2004; 51: 143-50.

6. Safar P, Escarraga LA, Chang F. Upper airway obstruction in the unconscious patient. J Appl Physiol 1959 14: 760-4.

7. Adnet F, Baud F. Relation between Glasgow Coma Scale and aspiration pneumonia. Lancet 1996; 348 (9020): 123-4.

8. Como JJ, Sutton ER, McCunn M, Dutton RP, Johnson $\mathrm{SB}$, Aarabi B, et al. Characterizing the need for mechani- 
cal ventilation following cervical spinal cord injury with neurologic deficit. J Trauma 2005; 59: 912-6.

9. Lu K, Lee TC, Liang CL, et al. Delayed apnea in patients with mid- to lower cervical spinal cord injury. Spine 2000; 25: 1332-8.

10. Stahel PF, Smith W, Moore E. Hypoxia and hypotension the "lethal duo" in traumatic brain injury: implication for prehospital care. Intensive Care Med 2008; 34: 402-4.

11. Gandía-Martínez F, Martínez-Gil I, Andaluz-Ojeda D, Bobillo de Lamo F, Parra-Morais L, Díez-Gutiérrez F. Analysis of early tracheostomy and its impact on development of pneumonia, use of resources and mortality in neurocritically ill patients. Neurocirugia (Astur) 2010; 21: 211-21.

12. Deem S. Management of acute brain injury and associated respiratory issues. Respir Care 2006; 51: 357-67.

13. Stocchetti N, Maas AI, Chieregato A, van der Plas AA. Hyperventilation in head injury: a review. Chest 2005; 127: 1812-27.

14. Kontos HA, Raper AJ, Paterson JL. Analysis of vasoactivity of focal $\mathrm{pH}, \mathrm{PaCO}_{2}$ and bicarbonate on pial vessels. Stroke 1977; 8: 358-60.

15. Fortune JB, Feustel PJ, de Luna C, Graca L, Hasselbarth J, Kupinski AM. Cerebral blood flow and blood volume in response to $\mathrm{O}_{2}$ and $\mathrm{CO}_{2}$ changes in normal humans. J Trauma 1995; 39: 463-71.

16. Bouma GJ, Muizelaar JP, Choi SC, Newlon PG, Young HF. Cerebral circulation and metabolism after severe traumatic brain injury: the elusive role of ischemia. J Neurosurg. 1991; 75: 685-93.

17. Muizelaar JP, van der Poel HG, Li ZC, Kontos HA, Levasseur JE, et al. Pial arteriolar vessel diameter and CO2 reactivity during prolonged hyperventilation in the rabbit. J Neurosurg 1988; 69: 923-7.

18. Coles JP, Fryer TD, Coleman MR, Smielewski P, Gupta AK, Minhas PS, et al. Hyperventilation following head injury: effects on ischemic burden and cerebral oxidative metabolism. Crit Care Med 2007; 35: 568-78.

19. Hutchinson PJ, Gupta AK, Fryer TF, Al-Rawi PG, Chatfield DA, Coles JP, et al. Correlation between cerebral blood flow, substrate delivery, and metabolism in head injury: a combined microdialysis and triple oxygen positron emission tomography study. J Cerb Blood Flow Metab 2002; 22: 735-45.

20. Carmona JA, Maas AIR, van der Brink WA, van Santbrink $\mathrm{H}$, Steyerberg EW, Avezaat CJJ. $\mathrm{CO}_{2}$ reactivity and brain oxygen pressure monitoring in severe head injury. Crit Care Med 2000; 28: 3268-74.

21. Muizelaar JP, Marmarou A, Ward JD, Kontos HA, Choi SC, Becker DP, et al. Adverse effects of prolonged hyperventilationin patients with severe head injury: a randomized clinical trial. J Neurosurg 1991; 75: 731-9.

22. Dumont TM, Visioni A, Rughani AI, Tranmer BI, Crookes B. Inappropriate Prehospital Ventilation in Severe Traumatic Brain Injury Increases In-Hospital Mortality. J Neurotrauma 2010; 27: 1233-41.

23. Roberts I. Hyperventilation therapy for acute traumatic brain injury. Cochrane Database of Systematic Reviews $2005 ; 3$.

24. The Brain Trauma Foundation. The American Association of Neurological Surgeons. The Joint Section on Neurotrauma and Critical are. Hyperventilation. J Neurotrauma 2000; 17: 513-20.

25. Dos Santos CC, Slutsky S. Cellular Responses to Mechanical Stress Invited Review: Mechanisms of ventilatorinduced lung injury: a perspective. J Appl Physiol 2000; 89: 1645-55.

26. Dreyfuss D, Soler P, Basset G, Saumon G. High inflation pressure pulmonary edema: respective effects of high airway pressure, high tidal volume and positive end-expiratory pressure. Am Rev Respir Dis 1988; 137: 1159-64.

27. Dreyfuss D, Saumon G. Ventilator-induced lung injury: Lessons from experimental studies. Am J Respir Crit Care Med 1998; 157: 294-323.

28. Mead J, Takishima T, Leith D. Stress distribution in lungs: a model of pulmonary elasticity. J Appl Physiol 1970; 28: 596-608.

29. Rouby JJ. Lung overinflation: the hidden face of alveolar recruitment. Anesthesiology 2003; 99: 2-4.

30. Muscedere JG, Mullen JBM, Gan K, Slutsky AS. Tidal ventilation at low airway pressure can augment lung injury. Am J Respir Crit Care Med 1994; 149: 1327-34.

31. Halter JM, Steinberg JM, Schiller HJ, Da Silva M, Gatto LA, Landas S, et al. Positive End-Expiratory Pressure after a Recruitment Maneuver Prevents Both Alveolar Collapse and Recruitment/Derecruitment Am J Respir Crit Care Med 2003; 167: 1620-6.

32. Webb HH, Tierney DF. Experimental pulmonary edema due to intermittent positive pressure ventilation with high inflation pressure: protection by positive end-expiratory pressure. Am Rev Respir Dis 1974; 110: 556-65.

33. Slutsky AS, Tremblay LN. Multiple System Organ Failure. Is Mechanical Ventilation a Contributing Factor? Am J Respir Crit Care Med 1998; 157: 1721-25.

34. Lowe GJ, Ferguson ND. Lung-protective ventilation in neurosurgical patients. Curr Opin Crit Care 2006; 12: 3-7.

35. Mascia L, Grasso S, Fiore T, Bruno F, Berardino M, Ducati A. Cerebro-pulmonary interactions during the application of low levels of positive end-expiratory pressure. Intensive Care Med 2005; 31: 373-9. 
36. Caricato A, Conti G, Della Corte F, Mancino A, Santilli F, Sandroni C. Effects of PEEP on the intracraneal system of patients with head injury and subarachnoid hemorrhage: the role of respiratory system compliance. J Trauma 2005; 58: 571-6.

37. López-Aguilar J, Villagrá A, Bernabé F, Murias G, Piacentini E, Real J. Massive brain injury enhances lung damage in an isolated lung model of ventilator-induced lung injury. Crit Care Med 2005; 33: 1077-83.

38. Mascia L, Zavala E, Bosma K, Pasero D, Decaroli D, Andrews $\mathrm{P}$, et al. High tidal volume is associated with the development of acute lung injury after severe brain injury: An international observational study. Crit Care Med 2007; 35: 1815-20.

39. The Acute Respiratory Distress Syndrome Network. Ventilation with lower tidal volumes as compared with traditional tidal volumes for acute lung injury and the acute respiratory distress syndrome. N Engl J Med 2000; 342: 1301-08.

40. Wolf S, Schürer L, Trost HA, Lumenta CB. The safety of the open lung approach in neurosurgical patients. Acta Neurochir Suppl 2002; 81: 99-101.

41. Holland MC, Mackersie RC, Morabito D, Campbell AR, Kivett VA, Patel R. The development of acute lung injury is associated with worse neurologic outcome in patients with severe traumatic brain injury. J Trauma 2003; 55: 106-11.

42. Robertson CS, Valadka AB, Hannay HJ, Contant CF, Gopinath SP, Cormio M, et al. Adult respiratory distress syndrome: A complication of induced hypertension after severe head injury. J Neurosurg 1999; 95: 560-8.

43. Bratton SL, Davis RL. Acute lung injury in isolated traumatic brain injury. Neurosurgery 1997; 40: 707-12.

44. Brower RG, Lanken PN, MacIntyre N, Matthay MA, Morris A, Ancukiewicz M, et al. National Heart Lung and Blood Institute ARDS Clinical Trials Network: Higher versus lower positive end expiratory pressures in patients with the acute respiratory distress syndrome. N Engl J Med 2004; 351: 327-36.

45. Gajic O, Frutos-Vivar F, Esteban A, Hubmayr RD, Anzueto A. Ventilator settings as a risk factor for acute respiratory distress syndrome in mechanically ventilated patients. Intensive Care Med 2005; 31: 922-6.

46. Tomicic V, Espinoza M, Andresen M, Molina J, Calvo $\mathrm{M}$, Ugarte $\mathrm{H}$, et al. Características de los pacientes que reciben ventilación mecánica en unidades de cuidados intensivos: primer estudio multicéntrico chileno. Rev Med Chile 2008; 136: 959-67.

47. Gattinoni L, Pelosi P, Vitale G, Pesenti A, D’Andrea L, Mascheroni D. Body position changes redistribute lung computed tomographic density in patients with acute respiratory failure. Anesthesiology 1991; 74: 15-23.

48. Pelosi P, Brazzi L, Gattinoni L. Prone position in acute respiratory distress syndrome. Eur Respir J 2002; 20: 1017-28

49. Alsaghir A, Martin, C. Effect of prone positioning in patients with acute respiratory distress syndrome: A meta-analysis. Crit Care Med 2008; 36: 603-9.

50. Beuret P, Carton MJ, Nourdine K, Kaaki M, Tramoni G, Ducreux JC. Prone position as prevention of lung injury in comatose patients: a prospective, randomized, controlled study. Intensive Care Med 2002; 28: 564-9.

51. Reinprecht A, Greher M, Wolfsberger S, Dietrich W, Illievich UM, Gruber A. Prone position in subarachnoid hemorrhage patients with acute respiratory distress syndrome: effects on cerebral tissue oxygenation and intracranial pressure. Crit Care Med 2003; 31:1831-8.

52. Thelandersson A, Cider A, Nellgard B. Prone position in mechanically ventilated patients with reduced intracranial compliance. Acta Anaesthesiol Scand 2006; 50: 937-41.

53. Chan KP, Stewart TE, Mehta S. High-frequency oscillatory ventilation for adult patients with ARDS. Chest 2007; 131: 1907-16.

54. Derdak S, Mehta S, Stewart TE, Smith T, Rogers M, Buchman TG, et al. High-frequency oscillatory ventilation for acute respiratory distress syndrome in adults: a randomized, controlled trial. Am J Respir Crit Care Med 2002; 166: 801-8.

55. Bollen CW, van Well GT, Sherry T, Beale RJ, Shah S, Findlay $\mathrm{G}$, et al. High frequency oscillatory ventilation compared with conventional mechanical ventilation in adult respiratory distress syndrome: a randomized controlled trial [ISRCTN24242669]. Crit Care 2005; 9: R430-9.

56. Salim A, Miller K, Dangleben D, Cipolle M, Pasquale M. High-frequency percussive ventilation: an alternative mode of ventilation for head-injured patients with adult respiratory distress syndrome. J Trauma 2004; 57: 542-6.

57. David M, Karmrodt J, Weiler N, Scholz A, Markstaller K, Eberle B, et al. High-frequency oscillatory ventilation in adults with traumatic brain injury and acute respiratory distress syndrome. Acta Anaesthesiol Scand 2005; 49(2): 209-14.

58. Hurst JM, Branson RD, Davis K Jr. High-frequency percussive ventilation in the management of elevated intracranial pressure. J Trauma 1988; 28: 1363-7.

59. Bein T, Kuhr LP, Metz C, Woertgen C, Philipp A, Taeger $\mathrm{K}$. ARDS and severe brain injury. Therapeutic strategies in conflict. Anaesthesist 2002; 51: 552-6. 